\title{
Salvage Reconstruction With the Masquelet Technique Following Wide Resection for Chondrosarcoma of the Proximal Femoral Metaphysis: A Case Report
}

\author{
MICHIYUKI HAKOZAKI ${ }^{1,2}$, RYOICHI KAWAKAMI ${ }^{1,3,4}$, NOBUYUKI SASAKI $^{1}$, \\ NARIHIRO TOSHIKI ${ }^{1}$, YOICHI KANEUCHI ${ }^{1}$, HITOSHI YAMADA ${ }^{1,5}$ and SHINICHI KONNO ${ }^{1}$ \\ ${ }^{1}$ Department of Orthopaedic Surgery, ${ }^{2}$ Higashi-Shirakawa Orthopaedic Academy, \\ ${ }^{3}$ Department of Traumatology and Reconstructive Surgery, and ${ }^{5}$ Department of Musculoskeletal \\ and Bone Metabolism, Fukushima Medical University School of Medicine, Fukushima, Japan; \\ ${ }^{4}$ Kawakami Orthopaedic Clinic, Fukushima, Japan
}

\begin{abstract}
Background: Bone reconstruction following a wide resection for a malignant musculoskeletal tumor remains challenging, especially for an intercalary defect following the resection of a metaphyseal lesion. Case Report: Here, we describe a surgical procedure using the Masquelet technique for the biological reconstruction of a huge subtrochanteric bone defect following failed pasteurized autologous bone grafting for a conventional chondrosarcoma of the proximal femoral metaphysis with a subtrochanteric pathological fracture. The patient, a 43-year-old Japanese male, was able to walk without a cane or a brace at 15 months after the final operation (International Society of Limb Salvage score, $86.7 \%$ ). Conclusion: This procedure should be considered as one of the reconstruction options following the wide resection of malignant bone tumors located in the metaphysis.
\end{abstract}

Bone reconstruction following a wide resection for a musculoskeletal malignancy is difficult and challenging. Surgical options are broadly classified into two groups: megaprosthetic reconstruction and biological reconstruction. A marked improvement of patients' vital prognoses by multidisciplinary treatment and biological reconstruction is in demand. However, when the biological reconstruction has

This article is freely accessible online.

Correspondence to: Dr. Michiyuki Hakozaki, Department of Orthopaedic Surgery, Fukushima Medical University School of Medicine, 1 Hikarigaoka, Fukushima-shi, Fukushima 960-1295, Japan. Tel: +81 245471276, Fax: +81 245485505, e-mail: paco@fmu.ac.jp

Key Words: Masquelet technique, induced membrane, biological reconstruction, malignant bone tumor, huge bone defect, metaphysis, adult. failed, the re-reconstruction methods for the remaining bone defect are limited.

The 'Masquelet technique', also known as the 'induced membrane technique', is one of the surgical techniques used for reconstructing bone defects. This technique was originally reported by Masquelet et al. in 2000 (1) as a method for reconstructing bone defects in the chronic phase following trauma (e.g., septic non-union after an open fracture). Thereafter, the Masquelet technique has also been applied for the primary treatment of open fractures with a bone defect $(2$, 3). Apart from trauma cases, this technique has been applied for bone defects after the resection of malignant bone tumors, mainly in pediatric patients (4-7), but a small number of adult patients has also undergone this technique with favorable outcomes (8). The Masquelet procedure consists of two stages $(9,10)$. In the first-stage, surgery is performed and the affected bone is removed and the bone defect is filled with polymethylmethacrylate (PMMA) cement. In the secondstage, surgery is performed 6-8 weeks later and the induced membrane around the PMMA cement is incised and the PMMA cement is removed; autologous cancellous bone is grafted, and the induced membrane is sutured. Although this procedure requires two separate surgeries, the procedure itself is quite simple.

Here, we describe a surgical procedure using the Masquelet technique for the biological reconstruction of a huge subtrochanteric bone defect following failed pasteurized autologous bone grafting, in a case of conventional chondrosarcoma with a pathological fracture.

\section{Case Report}

A previously healthy 43-year-old Japanese man slipped and fell on frozen ground. He was transported to the emergency room of the primary hospital and diagnosed with a bone tumor with a left subtrochanteric pathological fracture. Since the 


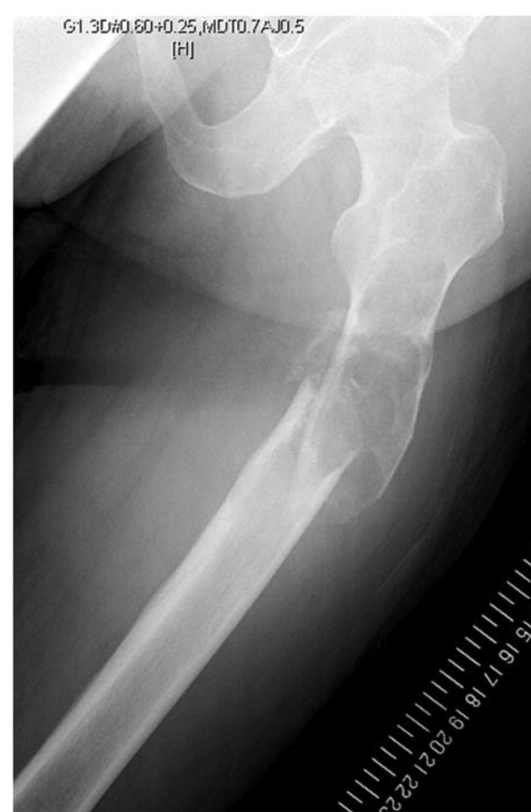

A

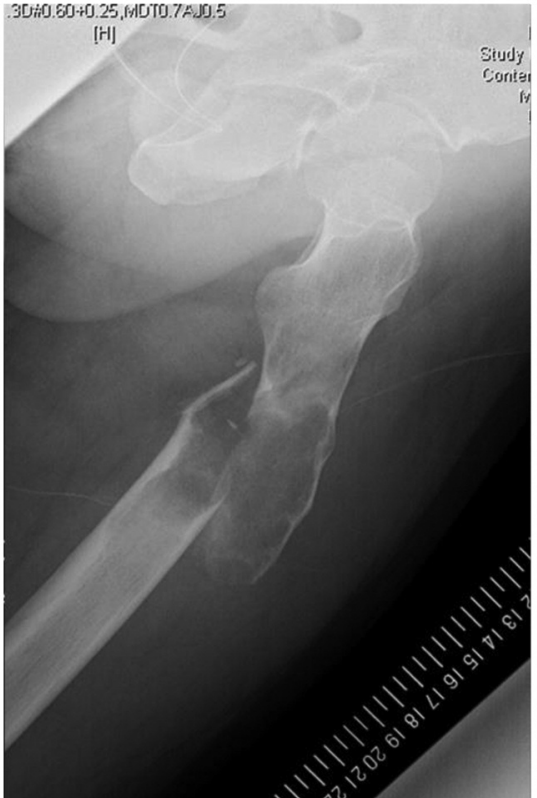

B

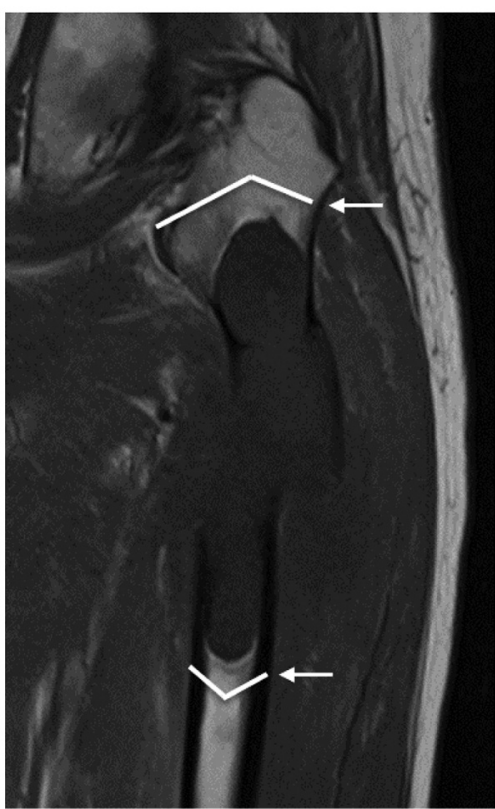

C

Figure 1. Initial plain radiography shows a metaphyseal osteolytic lesion with cortical ballooning and subtrochanteric pathological fracture on anteroposterior $(A)$ and lateral (B) imaging. Coronal T1-weighted MRI (C) reveals the intraosseous tumor without extracortical extension. Lines indicate the osteotomy line (arrows).

needle biopsy specimen was pathologically diagnosed as a low-grade conventional chondrosarcoma (grade 1), the patient was referred to our hospital. Plain radiographs revealed an osteolytic lesion with cortical ballooning on the left proximal femur and a subtrochanteric pathological fracture (Figure 1A, B). Magnetic resonance imaging revealed a bone tumor without extracortical extension, measuring approx. $140 \times 29 \times 31$ $\mathrm{mm}$ (Figure 1C). Chest computed tomography, as an additional radiological examination, revealed no other tumorous regions throughout the entire body.

Three weeks after the fracture, the patient underwent surgical treatment for the removal of the tumor. In the preoperative planning, we expected to encounter a $17-\mathrm{cm}$ bone defect after performing an en-bloc wide resection of the tumor and circumferential soft tissues. Although megaprosthetic reconstruction was also considered, we eventually selected pasteurized autologous bone grafting and bone fixation with an intramedullary interlocking nail and locking plate for the preservation of the patient's hip joint function (Figure 2).

The patient's lower extremity was kept non-weight-bearing and low-intensity pulsed ultrasound was also used; however, his grafted bone was gradually resorbed from 3 months after the operation (Figure 3). To preserve the patient's hip joint function, we decided to perform a biological reconstruction with the Masquelet technique after the removal of resorbing bone. Eight months after the operation, the resorbed bone was removed, and both the intramedullary interlocking nail and locking plate were left in place (Figure 4A, B). The huge 17-cm bone defect was filled with PMMA cement that contained $1 \mathrm{~g}$ of vancomycin per $40 \mathrm{~g}$ of PMMA cement (as part of the first-stage surgery) (Figure $4 C, D)$. At 8 weeks after the first-stage surgery, a second-stage surgery was performed as follows. We incised the induced membrane around the PMMA cement, carefully removed the PMMA cement, grafted autologous cancellous bone, and sutured the induced membrane. Although $155 \mathrm{~g}$ of autologous cancellous bone was harvested from the bilateral anterior and posterior iliac crest, it was not sufficient; thus, $56 \mathrm{~g}$ of artificial bone (betatricalcium phosphate, $\beta$-TCP) was additionally used (approx. 3:1 ratio) (Figure 5). After the final operation, consolidation was gradually observed (Figure 6), and full-weight bearing was permitted 6 months after the final operation.

The physical examination at 15 months after the final operation showed that the active range of motion of the patient's hip and knee were extension $20^{\circ}$ /flexion $120^{\circ}$ and extension $0^{\circ} /$ flexion $135^{\circ}$, respectively, and the extension and abduction muscular strength of the hip was evaluated as $5 / 5$ and $4 / 5$ in a manual muscle test, respectively. The patient was able to walk without a cane or a brace. Limb function was determined to be $86.7 \%$ according to the International Society of Limb Salvage (11). There was no indication of local or distant recurrence at 37 months after the first operation (tumor resection). 


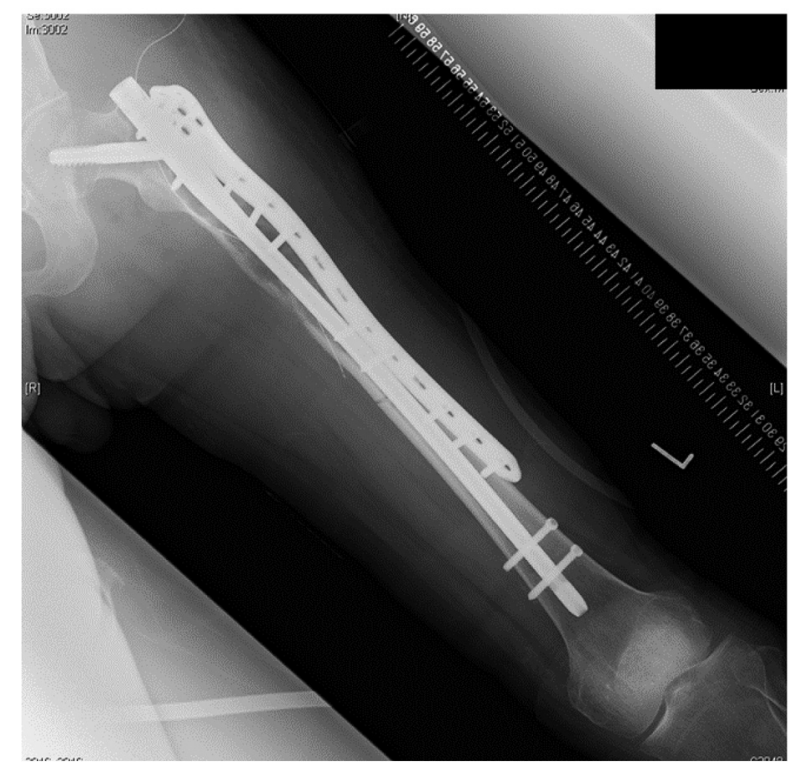

A

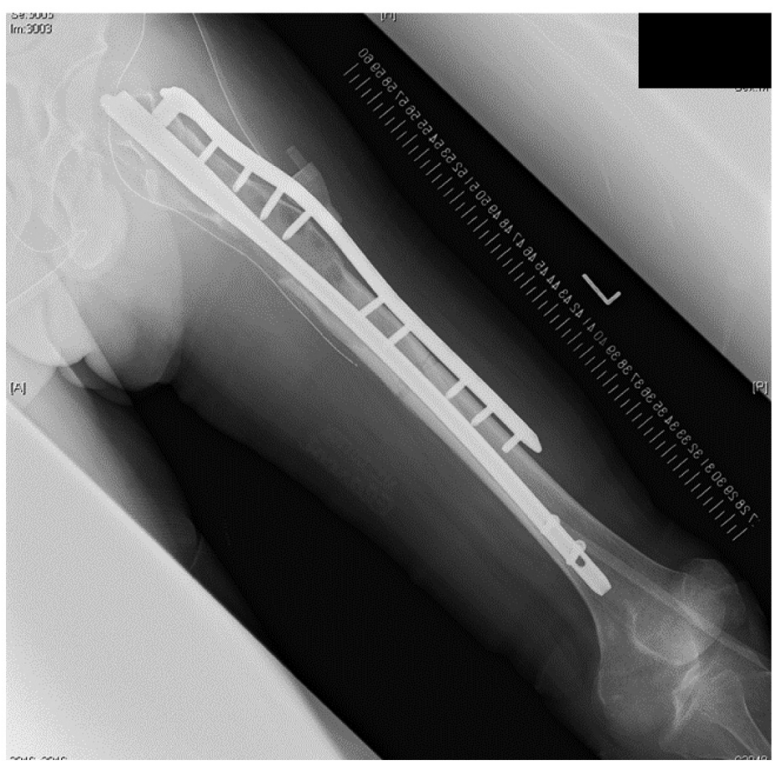

B

Figure 2. Plain radiography after the initial operation on anteroposterior (A) and lateral (B) imaging. After the wide resection and subsequent extracorporeal pasteurization, the treated autologous bone was fixed with an intramedullary interlocking nail and locking plate.

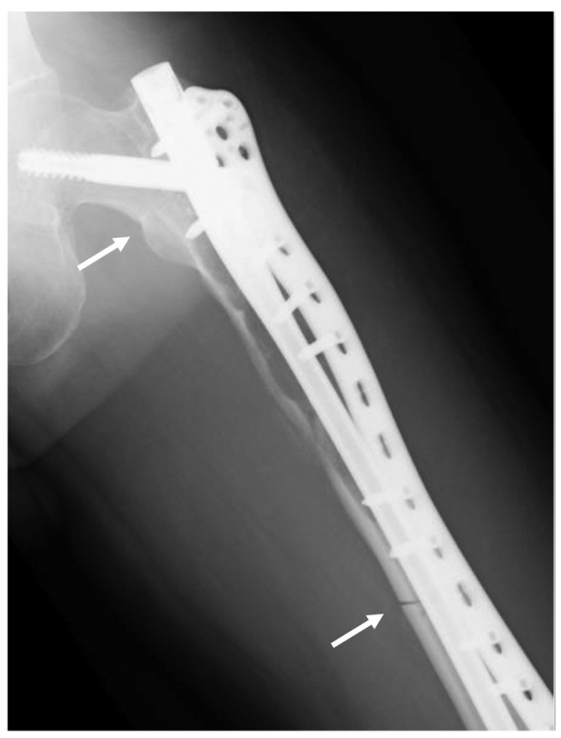

A

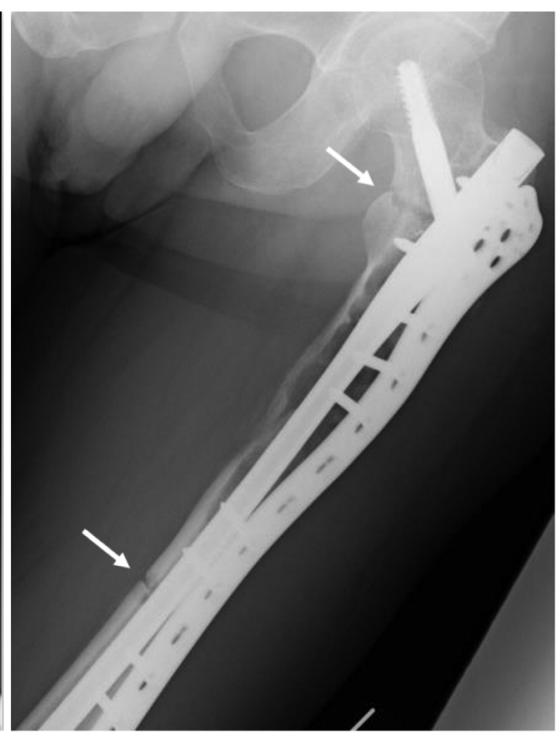

B

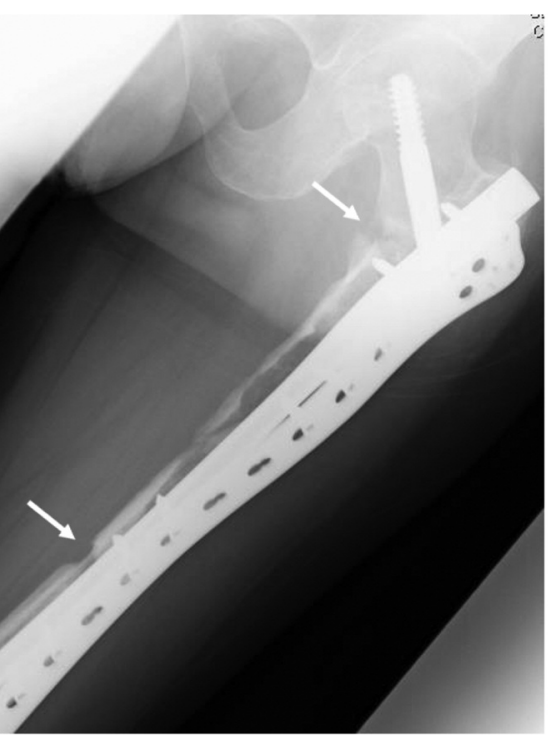

C

Figure 3. Anteroposterior plain radiography obtained at 1 (A), $3(B)$ and $7(C)$ months after the initial operation shows gradual resorption of the treated bone starting at 3 months after the surgery (arrows).

\section{Discussion}

Wide resection is the standard procedure for malignant bone tumors. The progress in multidisciplinary approaches has enabled salvaging of limbs in many cases. There are established procedures for reconstructing a bone defect after a wide resection. Megaprosthetic reconstruction is one of the most commonly used procedures, and it has provided 

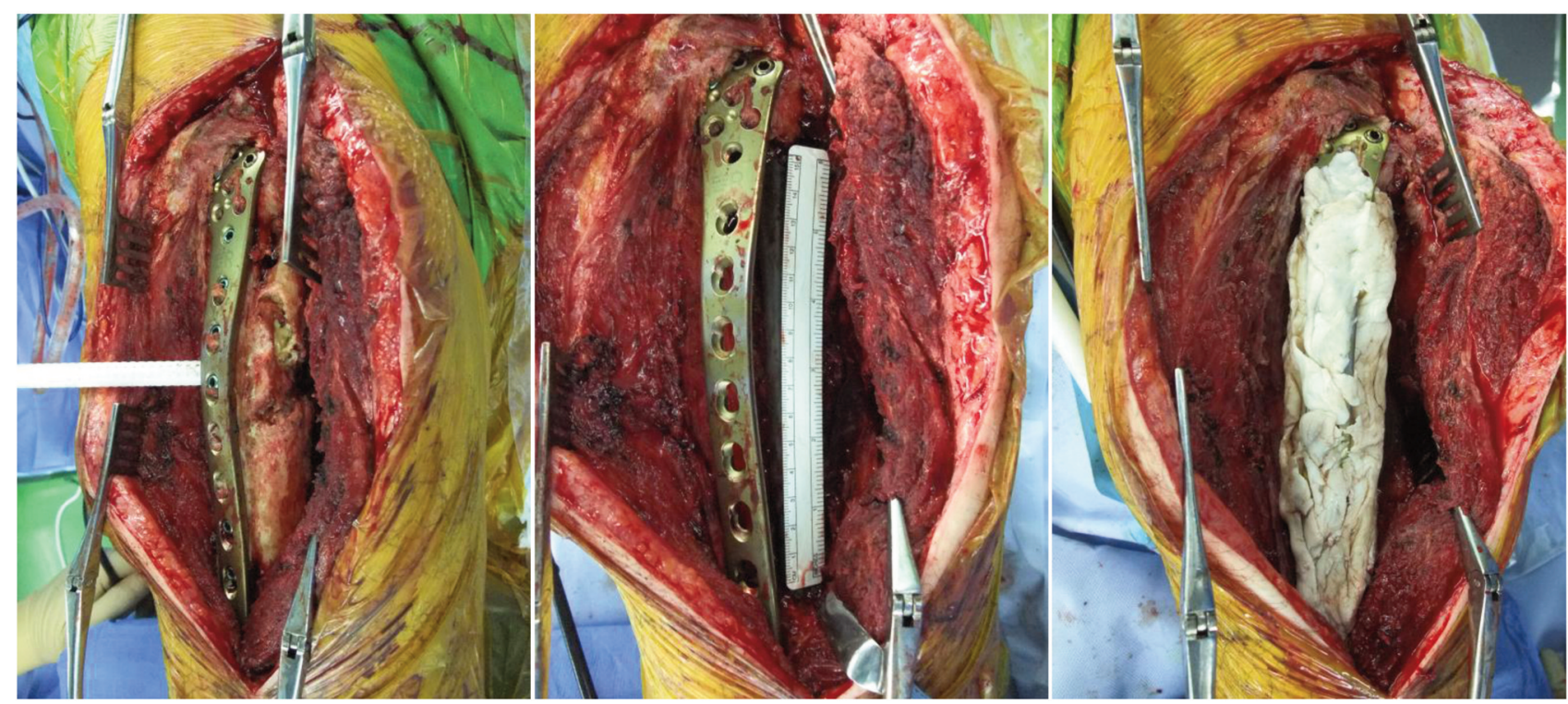

A

B

C

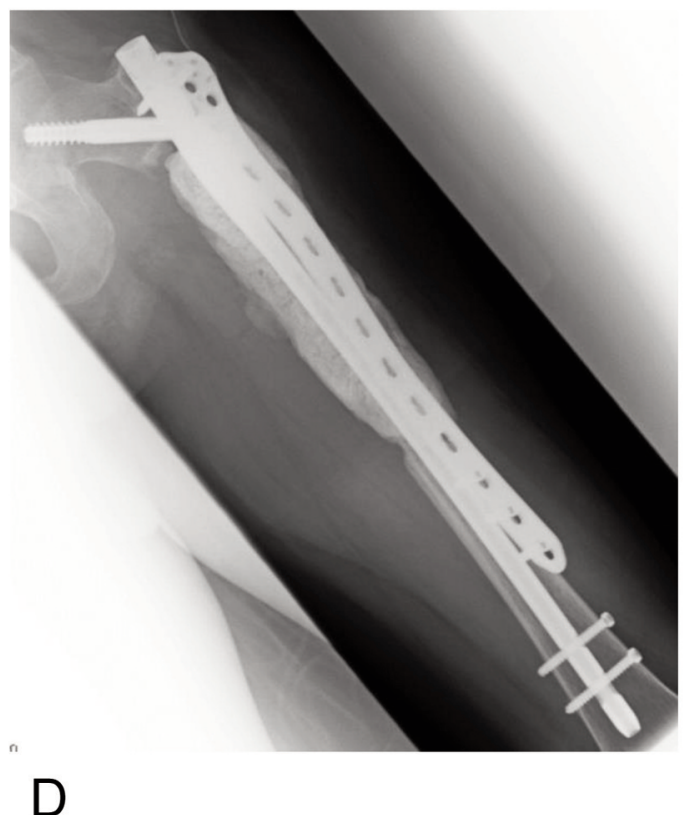

Figure 4. In the first-stage surgery using the Masquelet technique, absorbed bone (A) was removed and the 17-cm bone defect $(B)$ was filled with PMMA cement $(C, D)$.

favorable treatment outcomes. However, as a result of the marked improvement of patients' vital prognoses by multidisciplinary treatment, longstanding problems such as soft-tissue failure, aseptic loosening, implant breakage, periprosthetic fracture, and/or infection become unavoidable (12). In fact, a recent systematic review has indicated that the 10-year implant survival rate of proximal femoral megaprosthesis was $55 \%-86 \%$ (13). Other reconstruction procedures that can replace the use of megaprosthesis are thus required. The avoidance of megaprosthetic reconstruction for an intercalary bone defect after the resection of a metaphyseal lesion may allow preservation of the adjacent joint.

The reconstruction of an intercalary bone defect is another challenging problem. Although a segmental prosthesis is a possible surgical option, it cannot be applied 


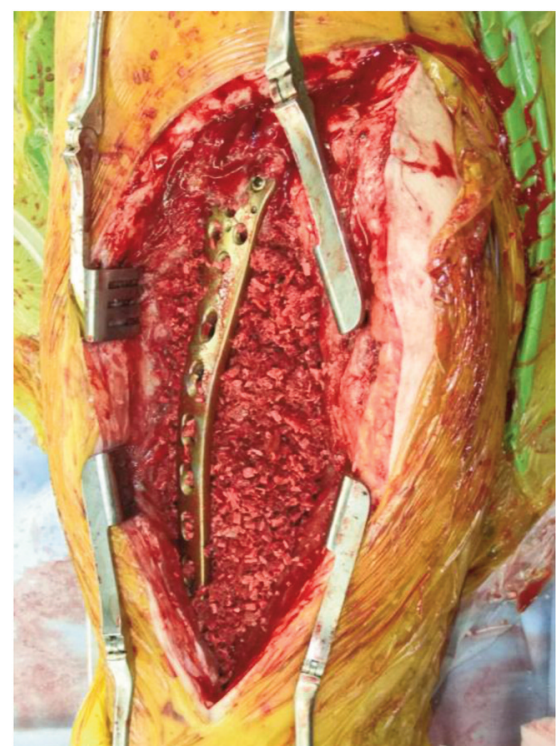

A

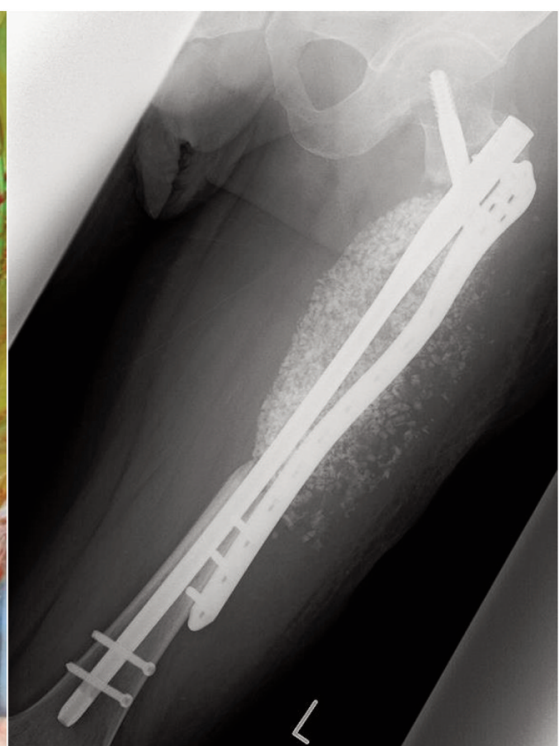

B

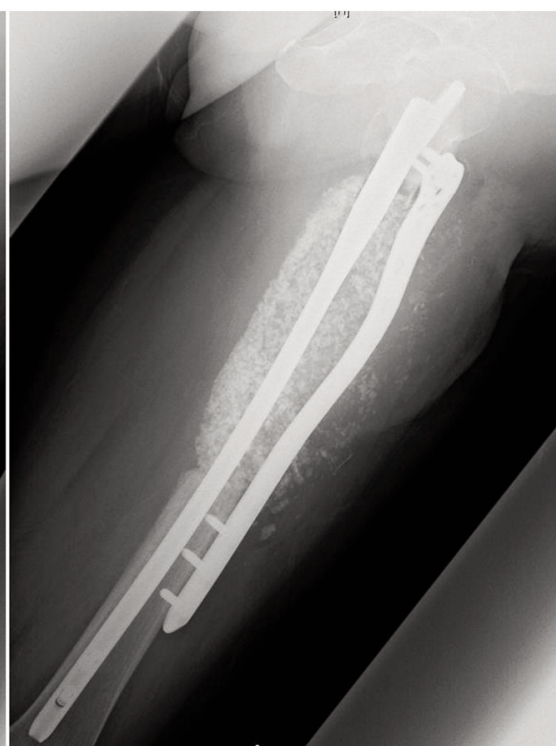

C

Figure 5. In the second-stage surgery, after the removal of the PMMA cement, the bone defect was filled with a mixture of autologous cancellous bone and artificial bone $(A-C)$.

for the metaphysis. The design of 3D-printed custom-made implants continues to advance (14). Biological reconstruction is now frequently used because of its favorable long-term functional outcome. Among the available biological reconstruction techniques, bulk allograft bone is globally used. However, in Asian countries including Japan, socio-religious reasons limit the use of allograft bone, and devitalized autologous tumor-bearing bone has been widely used. Nowadays, irradiation, pasteurization, or freezing with liquid nitrogen are used as devitalizing methods for tumor-bearing autologous bone in clinical settings (15). Other biological reconstruction procedures such as a free vascularized fibular graft or segmental bone transportation (distraction osteogenesis) are also available (15), but these are complicated surgical procedures, and there are limits on both surgeons and medical institutions. The clinical outcomes of treated autologous bone grafting have been reported to be favorable, but some types of failure such as nonunion in the junction, graft fracture, bone absorption, or infection may occur before the revitalization and maturation (15). In the failed cases, re-reconstruction methods after removing the failed treated autologous bone are limited. Although distraction osteogenesis or free vascularized bone grafting (e.g., with the fibula) are used for the biological reconstruction in a limited number of cases, megaprosthetic reconstruction (or sometimes amputation/disarthrocation) is selected in most cases.
In our patient's case, it was possible the resected bone to be used for treated autologous tumor-bearing bone grafting because of its advanced osteolysis and pathological fracture (15). However, we had plotted the therapeutic strategy considering the preservation of hip joint function as a top priority. Since bone resorption had gradually advanced as a result, we continued this therapeutic strategy. Intercalary biological reconstruction with the Masquelet technique was finally performed.

It was originally reported that the Masquelet technique can be indicated for huge bone defects, although conventional autologous bone grafting for diaphyseal bone defects $>6 \mathrm{~cm}$ may lead to incomplete healing (9). Kargar et al. have reported that the bone union success rate was not affected by the length of bone defects (16). However, in our institutional experience, the total amount of autologous cancellous bone harvested from the bilateral anterior and posterior iliac crest is limited, and it can be used only for segmental bone defects $<6 \mathrm{~cm}$ (3). Therefore, in the present case, we used $\beta$-TCP together with the harvested autologous cancellous bone as a bone substitute $(9,17,18)$ at an approx. 3:1 ratio for the $17-\mathrm{cm}$ bone defect, and good consolidation was eventually achieved.

In cases treated with autologous bone grafting for the reconstruction of a bone defect, rigid internal fixation with multiple fracture fixation devices are recommended (19). In our experience, when re-reconstructing failed-treated autologous bone grafting, the preservation of such internal fixation devices may make it easier to maintain the alignment, rotation, and length of the affected limb (10). 

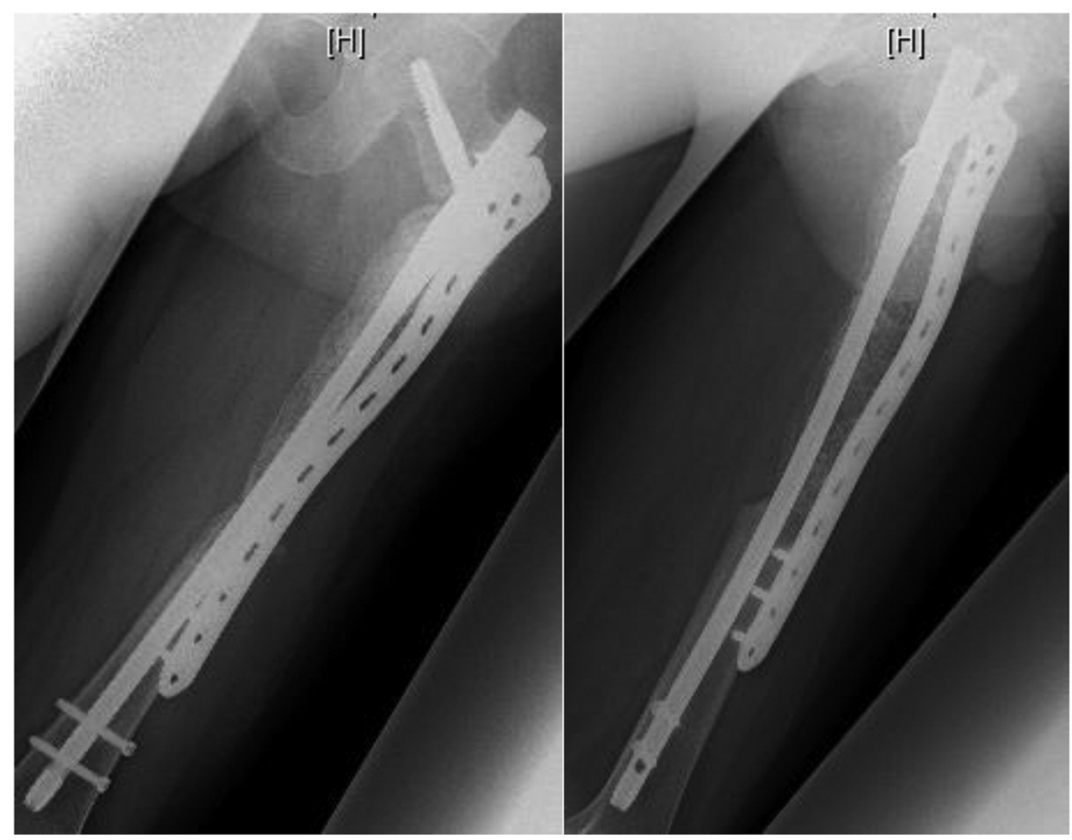

A

B
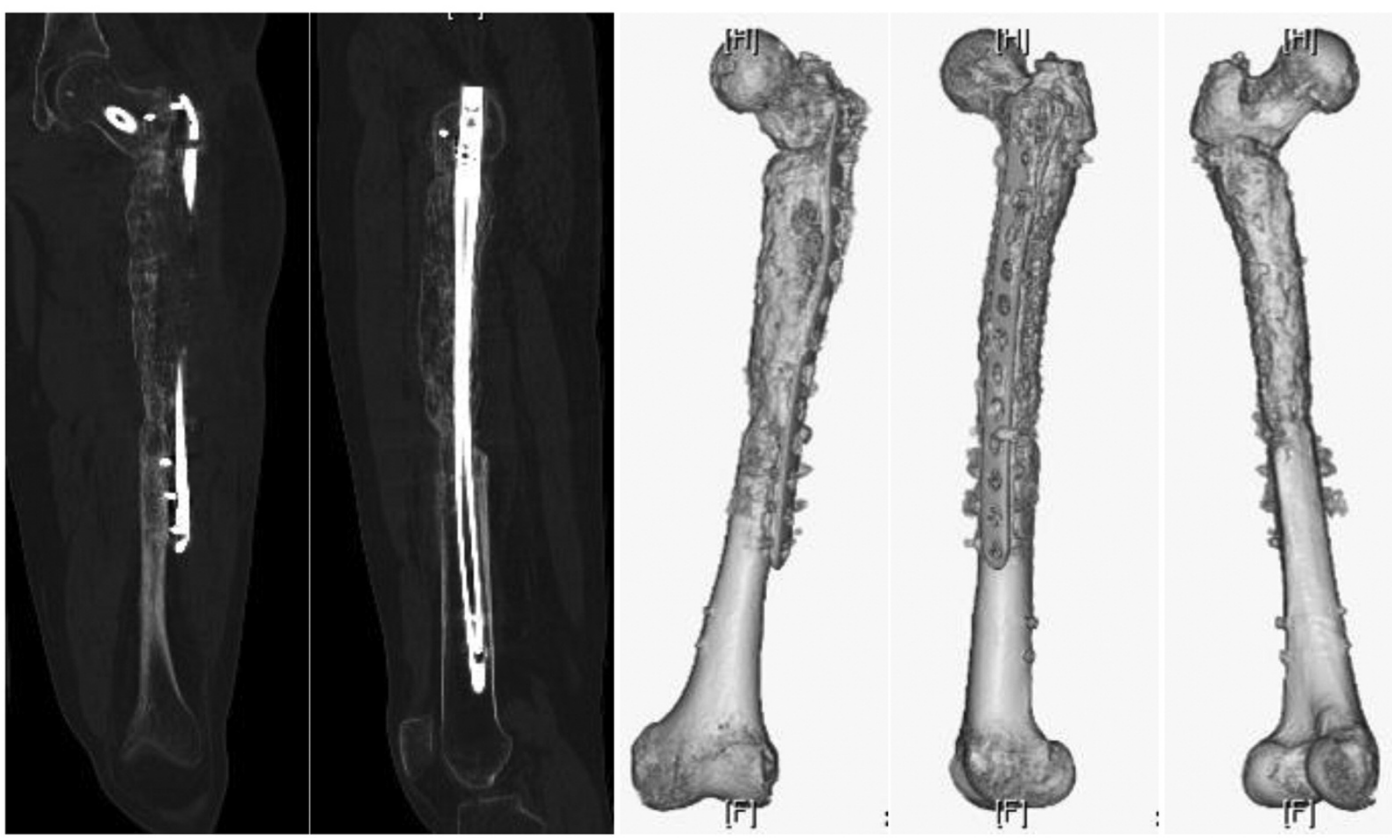

C

D

E

Figure 6. Anteroposterior (A) and lateral (B) plain radiography and coronal $(C)$, sagittal $(D)$ and $3 D(E) C T$ images obtained 1 year after the final operation show bone consolidation for the most part.

In conclusion, the Masquelet technique is a recommendable biological reconstruction method for salvaging failed-treated autologous tumor-bearing bone grafting as well as for the primary bone defect after a wide resection for a malignant bone tumor.

\section{Conflicts of Interest}

The Authors state that they have no conflicts of interest to declare in regard to this study. 


\section{Authors' Contributions}

MH operated the patient and wrote the manuscript. RK, NS, NT, YK, HY and SK were involved in a patient care, manuscript preparation and review. All Authors read and approved the final manuscript.

\section{References}

1 Masquelet AC, Fitoussi $\mathrm{F}$, Begue $\mathrm{T}$ and Muller GP: Reconstruction of the long bones by the induced membrane and spongy autograft. Ann Chir Plast Esthet 45: 346-353, 2000. PMID: 10929461.

2 Ronga M, Ferraro S, Fagetti A, Cherubino M, Valdatta L and Cherubino P: Masquelet technique for the treatment of a severe acute tibial bone loss. Injury 45: S111-115, 2014. PMID: 25457329. DOI: 10.1016/j.injury.2014.10.033

3 Hatashita S, Kawakami R, Ejiri S, Sasaki N, Toshiki N, Ito M, Konno SI and Hakozaki M: 'Acute Masquelet technique' for reconstructing bone defects of an open lower limb fracture. Eur J Trauma Emerg Surg, 2020. PMID: 31894350. DOI: 10.1007/s00068-019-01291-2

4 Biau DJ, Pannier S, Masquelet AC and Glorion C: Case report: Reconstruction of a 16-cm diaphyseal defect after Ewing's resection in a child. Clin Orthop Relat Res 467: 572-577, 2009. PMID: 19009325. DOI: 10.1007/s11999-008-0605-9

5 Villemagne T, Bonnard C, Accadbled F, L'kaissi M, de Billy B and Sales de Gauzy J: Intercalary segmental reconstruction of long bones after malignant bone tumor resection using primary methyl methacrylate cement spacer interposition and secondary bone grafting: The induced membrane technique. J Pediatr Orthop 31: 570-576, 2011. PMID: 21654468. DOI: 10.1097/BPO.0b013e31821ffa82

6 Accadbled F, Mazeau P, Chotel F, Cottalorda J, Sales de Gauzy $\mathrm{J}$ and Kohler R: Induced-membrane femur reconstruction after resection of bone malignancies: Three cases of massive graft resorption in children. Orthop Traumatol Surg Res 99: 479-483, 2013. PMID: 23608487. DOI: 10.1016/j.otsr.2013.01.008

7 Mansour TM and Ghanem IB: Preliminary results of the induced membrane technique for the reconstruction of large bone defects. J Pediatr Orthop 37: e67-74, 2017. PMID: 26469687. DOI: 10.1097/BPO.0000000000000663

8 Moteki T, Yanagawa T and Saito K: Autograft treated with liquid nitrogen combined with the modified Masquelet technique for bone defect after resection of malignant bone tumors: Two case reports. J Orthop Sci 24: 573-577, 2019. PMID: 28153375. DOI: 10.1016/j.jos.2017.01.001

9 Masquelet $\mathrm{AC}$ and Begue T: The concept of induced membrane for reconstruction of long bone defects. Orthop Clin North Am 41: 27 37, 2010. PMID: 19931050. DOI: 10.1016/j.ocl.2009.07.011

10 Han W, Shen J, Wu H, Yu S, Fu J and Xie Z: Induced membrane technique: Advances in the management of bone defects. Int $\mathrm{J}$ Surg 42: 110-116, 2017. PMID: 28478316. DOI: 10.1016/ j.ijsu.2017.04.064
11 Enneking WF, Dunham W, Gebhardt MC, Malawar M and Pritchard DJ: A system for the functional evaluation of reconstructive procedures after surgical treatment of tumors of the musculoskeletal system. Clin Orthop Relat Res 286: 241246, 1993. PMID: 8425352.

12 Capanna R, Scoccianti G, Frenos F, Vilardi A, Beltrami G and Campanacci DA: What was the survival of megaprostheses in lower limb reconstructions after tumor resections? Clin Orthop Relat Res 473: 820-830, 2015. PMID: 24964884. DOI: 10.1007/s11999-014-3736-1

13 Janssen SJ, Langerhuizen DWG, Schwab JH and Bramer JAM: Outcome after reconstruction of proximal femoral tumors: A systematic review. J Surg Oncol 119: 120-129, 2019. PMID: 30466190. DOI: $10.1002 /$ jso. 25297

14 Shehadeh AM, Isleem U, Abdelal S, Salameh H and Abdelhalim M: Surgical technique and outcome of custom joint-sparing endoprosthesis as a reconstructive modality in juxta-articular bone sarcoma. J Oncol 2019: 9417284, 2019. PMID: 32082379. DOI: $10.1155 / 2019 / 9417284$

15 Zekry KM, Yamamoto N, Hayashi K, Takeuchi A, Alkhooly AZA, Abd-Elfattah AS, Elsaid ANS, Ahmed AR and Tsuchiya H: Reconstruction of intercalary bone defect after resection of malignant bone tumor. J Orthop Surg (Hong Kong) 27: 2309499019832970, 2019. PMID: 30879390. DOI: 10.1177/230949 9019832970

16 Karger C, Kishi T, Schneider L, Fitoussi F, Masquelet AC and French Society of Orthopaedic Surgery and Traumatology (SoFCOT): Treatment of posttraumatic bone defects by the induced membrane technique. Orthop Traumatol Surg Res 98: 97102, 2012. PMID: 22244249. DOI: 10.1016/j.otsr.2011.11.001

17 Sasaki G, Watanabe Y, Miyamoto W, Yasui Y, Morimoto S and Kawano H: Induced membrane technique using beta-tricalcium phosphate for reconstruction of femoral and tibial segmental bone loss due to infection: Technical tips and preliminary clinical results. Int Orthop 42: 17-24, 2018. PMID: 28536801. DOI: $10.1007 / \mathrm{s} 00264-017-3503-5$

18 Gupta S, Malhotra A, Jindal R, Garg SK, Kansay R and Mittal $\mathrm{N}$ : Role of beta tri-calcium phosphate-based composite ceramic as bone-graft expander in Masquelet's-induced membrane technique. Indian J Orthop 53: 63-69, 2019. PMID: 30905983. DOI: 10.4103/ortho.IJOrtho_240_17

19 Lee SY, Jeon DG, Cho WH, Song WS and Kim BS: Are pasteurized autografts durable for reconstructions after bone tumor resections? Clin Orthop Relat Res 476: 1728-1737, 2018. PMID: 30794210. DOI: 10.1007/s11999.0000000000000100

Received June 30, 2020

Revised August 4, 2020

Accepted August 5, 2020 